\title{
ROCAS BLANDAS Y RIESGOS EN EL SUR DE LA COMUNIDAD VALENCIANA (ESPAÑA)
}

\author{
Juan Antonio Marco Molina
}

\section{RESUMEN}

La evidente relación entre rocas blandas y ciertos fenómenos erosivos que implican cierta peligrosidad, así como las intervenciones humanas que actúan como factor de inestabilización de las laderas, con la génesis de formas de vaciado (cárcavas, gullies, ravines y procesos de piping) y deslizamientos, son los elementos que se tienen en cuenta como factores determinantes de un riesgo, que es preciso considerar en tareas de ordenación del territorio a escala municipal o supramunicipal.

\begin{abstract}
The evident relation between soft rocks and some erosive phenomenons which implicate certain riskiness, as soon as human interventions which act as an inestability factor of slopes, with the genesis of a highly dissected landscape (badlands, gullies, ravines and piping) and slumps, are the elements borne in mind as determinant factors of a hazard, which is essential to considerate for the territorial management in the municipal or supramunicipal scales.
\end{abstract}

Key words: hazard, management, badlands, slumps, land use.

El objetivo de este trabajo, como aproximación al tema indicado en el título, es señalar, desde un punto de vista cualitativo, la incidencia de ciertos procesos de ladera en su relación con la dinámica actual de las mismas y con ciertas intervenciones antrópicas, es decir, con una perspectiva geográfica.

Por otra parte, como simple introducción, es preciso acotar una parcela concreta dentro de una temática tan amplia como es la de la Geografía y los riesgos (CALVO, 1984). En este caso se han considerado exclusivamente los fenómenos relacionados con litofacies o formaciones superficiales blandas que, de forma inherente, implican cierta peligrosidad; peligrosidad que deriva no sólo de las características físicas de las mismas, sino, sobre todo, de los rasgos climáticos condignos del ámbito estudiado. Básicamente, dichos rasgos son: por un lado, la acusada sequía estival que actúa como factor de preparación del material, y por otro, las precipitaciones de elevada intensidad y carácter torrencial. Sin 
embargo, lo que confiere un marcado carácter geográfico a esta cuestión es su relación con las formas de aprovechamiento u ocupación del suelo; relaciones referidas a la intervención antrópica tanto como factor desencadenante o acelerador de un fenómeno — se podría hablar de «impacto»-, como por las consecuencias negativas derivadas de la peligrosidad, es decir, el «riesgo».

Con todo, es preciso señalar la importancia de las litofacies blandas y formaciones superficiales en la organización del territorio, así como los fenómenos asociados a las mismas; de manera que posteriormente se puedan relacionar dichos procesos con determinadas intervenciones antrópicas y sus efectos, especialmente en dos ejemplos concretos; todo ello en el ámbito de la provincia de Alicante.

\section{Distribución de rocas blandas y formaciones superficiales, y su importancia en la organización tradicional del espacio}

Con la denominación genérica de rocas blandas se engloban materiales de edad muy diversa, cuyo rasgo común es la deleznabilidad y escasa coherencia, de ahí que se incluyan también los materiales detríticos; son pues las vertientes constituidas por margas de distinta edad (Keüper, Albiense, Paleóceno, Oligoceno, Mioceno, entre otros) y los depósitos arcillosos y detríticos cuaternarios, los que llaman nuestra atención, puesto que han merecido una valoración muy positiva en el proceso de ocupación del territorio, al tiempo que implican cierta peligrosidad, y por tanto, potencialidad de riesgo.

En efecto, el primero de estos aspectos viene determinado por el modo en que se distribuyen dichos materiales fruto de la compartimentación estructural del relieve, puesto que son las unidades estructuralmente deprimidas — grandes fosas-, las áreas en las que abundan los materiales detríticos cuaternarios o los materiales margosos; de tal manera que los ámbitos preferentes de la agricultura han sido las vegas de los ríos, superficies de glacis y abanicos aluviales, caracterizando el paisaje de amplios espacios: Vega y Campo del Segura, Camp d'Elx, Camp d'Alacant, valles del Vinalopó y llanos de la Marina y el Marquesat de Dénia, y, todo ello a pesar de que en algunos casos, las unidades morfológicas presenten serios inconvenientes derivados de la dinámica inherente a las mismas; siendo las inundaciones, en especial en la Vega del Segura, el principal riesgo, con una evidente contradicción entre la intensa ocupación del territorio y los procesos de crecida (CALVO, 1984).

Frente a estas áreas características del litoral y de zonas prelitorales, destaca un amplio espacio serrano donde, por lo anfractuoso del terreno, es muy acusada la relación entre las litofacies señaladas y los espacios agrícolas, hasta el punto de que hay un gran paralelismo entre los afloramientos de éstas y el desarrollo del espacio agrícola tradicional.

La agricultura únicamente tiene posibilidades en las siguientes unidades fisiográficas:

- los valles o depresiones tectónicas, constituidos comúnmente por margas miocenas, a veces fosilizadas por depósitos cuaternarios, y, por lo general, de pequeñas dimensiones — sólo destacan por su extensión la Foia d'Alcoi y la de Castalla-;

- los taludes margosos de las frecuentes crestas hasta donde la pendiente lo permite, con ejemplos paradigmáticos en los que los pasillos margosos de una serie alternante son los espacios aterrazados entre yermos calizos; al tiempo que también destacan los taludes integrados por depósitos de ladera;

- y, por último, las depresiones cársticas o zonas de acumulación de arcillas residuales propias del modelado de las calizas. 
Son espacios en los que, en general, y a excepción de los señalados en último lugar, las pendientes son acusadas y los llanos escasos y reducidos, de tal forma que la ocupación de este territorio tiene como base esencial el aterrazamiento, es decir, la regulación de las laderas con una doble finalidad: incrementar la infiltración y retener el terreno (MORALES, 1969 y 1989; MORALES y BOX, 1986).

La descripción de un sector reducido en el interior de uno de los macizos puede reflejar el aspecto señalado, sin olvidar que, en definitiva, se trata de una valoración del medio físico o complejo ecológico hecha por el hombre. El espacio elegido es la vertiente septentrional de Aitana, elección que está justificada porque allí están representadas todas las unidades fisiográficas señaladas. Es un sector en el que una estructura fallada ha fragmentado una porción de la cobertera sedimentaria que integran materiales muy contrastados (MARCO, 1990); de manera que desde el bloque culminante (1.558 m), conceptuado como horst-cresta, y hacia el norte, se suceden las calizas eocenas, margas verdosas del mismo período y la serie alternante predominantemente margosa del Paleóceno, mientras que al pie del mismo, entre 1.250 y $1.000 \mathrm{~m}$ de altitud, una compleja estructuras en escalera de fallas determina el hundimiento de los bloques eocenos hacia el norte, pero de forma que en el seno de la misma hay sectores en los que el Oligoceno aparece en pequeñas dovelas hundidas, en unos caso, a modo de pequeñas cubetas $\mathrm{y}$, en otros, como valles elevados, constituidos por la serie fundamentalmente margosa oligocena.

En este contexto, son las litofacies blandas señaladas, así como los depósitos de ladera al pie de los frecuentes escarpes de falla, los sectores que constituyen el espacio agrícola tradicional; sobre todo en la parte occidental de este escalera de fallas. En la parte oriental, los materiales oligocenos no afloran — todo el conjunto está basculado hacia el oeste-, y la escalera de fallas afecta casi de forma exclusiva a las calizas nummulíticas eocenas, a las que corresponde el dominio en el paisaje — morros y penyes -; no obstante, depósitos de ladera y, sobre todo, las cubetas cársticas rellenas de terra rossa — clots y foietes - tienen una significación muy importante en el paisaje como únicos espacios aptos para la agricultura. De hecho, la comparación entre cartografía existente, fotointerpretación y prospección en el terreno, evidencian estas relaciones.

\section{Fenómenos propios de estas litofacies}

Por su situación preferente en tramos altos o cabeceras son frecuentes las pendientes generales acusadas, así como las propias de los taludes de los cauces, muy ahocinados en margas o aluviones. Circunstancias que, junto a los rasgos climáticos señalados y la intensa deforestación que afecta a la mayor parte del territorio, determinan el predominio de los procesos de vaciado, de tal modo que cárcavas y ramblizos están presentes, y de forma muy extendida, en todo el ámbito provincial (fig. 1). Sin embargo, hay que precisar que se pueden distinguir áreas localizadas en cauces o proximidades de los mismos que son reflejo de los mecanismos morfológicos actuales o subactuales, de aquellas en las que estos procesos afectan a materiales en los que se marcan intervenciones antrópicas, muy a menudo, desencadenantes o aceleradoras de estos procesos erosivos. Es decir, se puede distinguir entre sectores morfológicamente «inestables» y los que sólo lo son «potencialmente», siendo el «impacto» el factor fundamental de la actualización del riesgo.

Por otra parte, estas litofacies, y en especial las margas, presentan un rasgo muy a tener en cuenta, pues son materiales expansivos; es decir, que, embebidos en agua, adquieren plasticidad y, con ello, experimentan movimientos de reptación o deslizamientos rotacionales, cuando no configuran auténticas coladas de fango. Estos procesos, además de 
Fото 1. Modelado habitual de las margas en taludes de cauces son las cárcavas (bad lands); margas senonienses en un afluente del Montnegre.

Fото 2. Deslizamientos rotacionales y coladas de fango en taludes margosos son procesos frecuentes en la Foia d'Alcoi; ejemplo situado al norte del Poble Nou de Sant Rafael, Cocentaina. 
FiguRA 1. Áreas acarcavadas en la provincia de Alicante (España) (rayado horizontal).

relacionarse con margas (neógenas, cretácicas o del Keüper), se localizan en sectores donde es importante la remoción basal o zapa lateral, es decir, en taludes o márgenes de cursos fluviales encajados en dichos materiales. Así mismo, rasgo común, aunque no exclusivo, en estos procesos o fenómenos es la acusada pendiente de la ladera y la morfología plana de la culminación de la misma. De manera que se robora una estrecha relación de estos movimientos de masa con cauces profundamente encajados en margas o arcillas, hasta el punto que se pueden señalar ejemplos en la cuenca del riu Montnegre en las margas miocenas de la Foia de Castalla; en las margas y arcillas del Keüper, en el riu Algar; pero, donde este tipo de fenómenos adquieren gran relevancia es en los cauces encajados en las margas miocenas tanto de la Foia d'Alcoi como de los valles periféricos a la misma (Vall de Ceta, Vall de Planes, entre otros), donde ha sido objeto de estudios 
monográficos (LA ROCA, 1990). Sin embargo, al igual que en el caso de los acarcavamientos, es preciso resaltar aquellas actuaciones antrópicas con las que se verifica un alto grado de riesgo de movimientos de masa. En definitiva, se puede aplicar el mismo esquema esbozado anteriormente de diferenciar áreas inestables y áreas potencialmente inestables.

De ahí que el conocimiento de las intervenciones — «impactos»- causantes de tales efectos sea de gran interés como referencia de diagnóstico para tener en cuenta la potencialidad de riesgo de erosión y deslizamiento en estudios de ordenación del territorio.

\section{Tipos de intervenciones antrópicas}

La actuación del hombre en cuanto percutor o acelerador de ciertos procesos que implican, en esta ocasión, serios riesgos de erosión o acarcavamiento y de deslizamiento, se relacionan con el aprovechamiento u ocupación del territorio, tanto porque se trate de nuevas actividades, como por tratarse de procesos de abandono o deterioro de sistemas tradicionales de aprovechamiento del suelo. Estos «mecanismos y procesos antrópicos de alteración» destacados ya en otra ocasión (VERA y MARCO, 1988), son fundamentalmente la desorganización de los sistemas tradicionales de aprovechamiento de las laderas subáridas, las nuevas transformaciones de ladera para cultivo, las repoblaciones forestales, infraestructuras de comunicaciones y remoción de terrenos para urbanizar; siendo precisamente sobre rocas blandas cuando suponen un mayor impacto ambiental que actualiza un riesgo potencial evidente.

Las diferentes actuaciones antrópicas señaladas se distribuyen de forma muy puntual y aislada en el territorio alicantino, de manera que un análisis pormenorizado de cada una de ellas se convertiría en un inventario casi inacabable. Por ello se han seleccionado dos sectores en los que de forma más o menos clara se sinteticen tanto los «impactos» como los «riesgos» señalados.

\subsection{El Palomaret}

Sector situado en el borde serrano septentrional del Camp d'Alacant que, en principio, podemos calificar como un espacio rural, que ha experimentado profundas mutaciones en los últimos treinta años, mutaciones relacionadas con cambios en los aprovechamientos del suelo que son manifiestos en el paisaje. Para su análisis se ha realizado la fotointerpretación del sector a partir de los positivos correspondientes a 1956, 1978 y 1985; así como las prospecciones de campo (VERA y MARCO, 1988). Del primero de los aspectos citados se desprende la siguiente evolución (fig. 2):

- En 1956 se observan los elementos propios del paisaje rural de la época, es decir, predominio de un aprovechamiento agrícola de secano con riego eventual de agua de avenida, donde se ha producido ya el abandono de las tierras más marginales. El resto del territorio está ocupado por el matorral.

— En 1978 se ha producido el abandono de la práctica totalidad de las tierras de cultivo, que han pasado de ocupar 84,5 ha a menos de 7 ha. Pero lo realmente interesante es la aparición de nuevas intervenciones: roturaciones para la plantación de pinos, preparación de espacios para urbanizar mediante desmontes y parcelaciones en terrazas, y la proliferación de caminos y carreteras.

- En 1985 el espacio acondicionado para urbanizar alcanza ya las 214,5 ha mientras que carreteras y caminos tienen aproximadamente $20 \mathrm{~km}$ de longitud. 
Figura 2. Croquis de las mutaciones paisajísticas del sector del Palomaret (Alicante, España). 
Fото 3. Explanaciones para urbanizar que conllevan desorganización de la red de avenamiento y concentración de escorrentías por cunetas, suelen acelerar, cuando no iniciar, intensos procesos de vaciado; ejemplo de cauce abierto a partir de una cuneta en explanación destinada a urbanizar, El Palomaret, Petrer.

Estas son las intervenciones antrópicas acaecidas sobre unas laderas cuya situación se debía calificar de cierta estabilidad en 1956, pues las formas de vaciado (cárcavas) se localizan en los cauces de barrancos y ramblas, mientras que el resto del espacio, entre la culminación de Penyes Monteses y el Palomaret propiamente dicho, constituido por la ritmita predominantemente margosa del Albiense y recubierta por depósitos de ladera encostrados que actuaban de coraza protectora de las margas infrayacentes, presentaba un claro aspecto de estabilidad.

De este modo, es posible establecer las relaciones entre cada una de las intervenciones y sus efectos: 
- el abandono de terrazas de cultivo propicia la aparición de cárcavas (ravins o rigoles) en sectores margosos y/o de fuertes pendientes, mientras que en espacios constituidos por aluviones y relleno, pendientes más suaves y/o en vaguadas (cañadas), los fenómenos más habituales son los de entubamiento o erosión en túnel (piping) que, una vez colapsados, evolucionan hacia ramblizos (gullies), en una relación señalada ya en otras cuencas (HARVEY, 1982). Si bien, es preciso reconocer la discontinuidad espacial de estos procesos, pues hay ejemplos de una rápida recuperación de la vegetación, o bien ésta sucede a los procesos erosivos. No obstante, los inconvenientes de esta desorganización no se limitan a estos graves riesgos de erosión, sino que influyen directamente en el incremento de los débitos líquidos y sólidos en cuencas vertientes y de los riesgos de inundación; aspectos señalado ya anteriormente en casos como el del barranco de las Ovejas (MORALES, BRU y BOX, 1983; MORALES y BOX, 1986; GIL et alt., 1983 y GIL et alt., 1986).

- las repoblaciones forestales: el efecto de inestabilización producida por los aterrazamientos se registra de forma bien patente en las colinas situadas al sur de la hacienda el Palomaret, en las que si bien en 1956 se marcan algunos ejemplos de cárcavas, en 1978, y sobre todo en 1985, son prácticamente generalizadas (vid. fig. 2). Procesos que han sido señalados en otros sectores de la provincia de Alicante (VERA Y MARCO, 1988) o en el Rif occidental (NEBOIT, 1983).

- desmontes y movimientos de tierra para urbanizar: se han encargado no sólo de inestabilizar la mayor parte de la ladera, sino que además han provocado una desorganización de la red de avenamiento. Los procesos originados son la profusión, no sólo de surcos (ravins o rigoles), sino de auténticas cárcavas, movimientos de masa, y la génesis de conos de derrubios. Realmente espectaculares resultan las interferencias en la red de avenamiento, pues los aplanamientos realizados al sur de la carretera de acceso al Palomaret (vid. fig. 2, b) eliminaron por completo el cauce de un barranco que, tras los episodios de lluvias de elevada intensidad de octubre de 1982 especialmente, se volvió a encajar (vid. fig. 2, c), evidenciando la elevada capacidad de arrastre de estos cursos de comportamiento torrencial.

- las infraestructuras de comunicaciones: en este caso, carreteras y caminos, suponen alteraciones muy similares a las anteriores que se manifiestan mediante formas y procesos idénticos; incluso, se han observado tubos verticales (pipes) semicolapsados en las calzadas, así como los efectos de las cunetas como elementos de concentración de la escorrentía y génesis de encajamientos que en ocasiones superan los dos metros de profundidad.

Son, en suma, actuaciones acometidas sin una valoración previa del medio físico, de manera que se asigna al territorio un tipo de aprovechamiento cuya intensidad y características supera las posibilidades de acogida y tolerancia del mismo.

\subsection{La Foia d'Alcoi}

La Foia d'Alcoi forma parte de un dominio morfoestructural del territorio prebético alicantino que en otras ocasiones se ha denominado franja cretácica septentrional (MARCO, 1990). Dicha unidad es solución de continuidad entre dos conjuntos situados a levante $\mathrm{y}$ poniente, en los que las unidades estructurales se disponen, fundamentalmente, de 
Fото 4. La alteración de los perfiles en los taludes margosos y arcillosos, determina la inestabilización de los mismos, con los consiguientes problemas de reptación y deslizamiento; ejemplo localizado en la carretera Cocentaina-Benilloba, Cocentaina.

suroeste a noreste, mientras que dicha Foia, fosa tectónica en realidad, dispuesta de SSW a NNE, mediante fallas, supone la interrupción de las sierras (horsts), de tal forma que los valles (depresiones tectónicas) pasan a ser, sin solución de continuidad que las diferencie, partes de la fosa propiamente dicha, con lo que las depresiones constituidas por margas miocenas se convierten en apéndices del sector más deprimido de la fosa, hacia el que se dirige la escorrentía.

La morfología, tanto de la fosa como de las depresiones periféricas, se debe esencialmente al proceso de instalación de la red de avenamiento que jerarquiza el riu Serpis. Es precisamente la instalación de la red la que confiere mayor singularidad a buena parte de las depresiones y a los terrenos margosos situados al este del Serpis. Allí, el encajamiento de barrancos y ríos a partir de un nivel de aplanamiento, depara la presencia de cauces muy ahocinados, con profundidades entre 80 y $90 \mathrm{~m}$, separados por estrechos interfluvios, llanos reducidos - plans y llomes - o cerros del citado nivel superior denominados generalmente tossals, tossalets o alts, configurando en principio un relieve ciertamente anfractuoso (fig. 3).

Son precisamente los rasgos señalados los que explican que en estos terrenos margosos, los taludes, tanto de cerros como de ríos y barrancos, presenten pendientes considerables; es decir, fuertes pendientes en materiales margosos (plásticos) de culminación plana, a menudo afectados en su base por corrientes fluviales. Son, en suma, los factores que determinan la existencia de deslizamientos, coladas de fango (solsides) o simplemente 
FIGURA 3. Bloques diagramas esquemáticos representativos de la Foia d'Alcoi del proceso de instalación de la red de asentamiento. 
desplomes, localizados en los susomentados taludes. Fenómenos cuyas principales características son un largo período de preparación y un desarrollo rápido; de manera que les es condigna una gran discontinuidad tanto espacial como temporal.

Se trata pues de unidades morfológicas, taludes margosos, que presentan en sí mismas un evidente grado de peligrosidad, inherente a la dinámica de este tipo de laderas, sobre todo allí donde un curso fluvial ejerce un decisivo papel de remoción basal, especialmente en momentos de crecida.

Sin embargo, estas unidades morfológicas entrañan un claro riesgo en el caso de que se produzca la ocupación antrópica de las mismas, situación de la que la ciudad de Alcoi es un buen ejemplo: la ocupación de la parte alta de los taludes ha obligado a la defensa y protección de los mismos en momento de avenida ante el «riesgo» de deslizamientos (COSTA y MATARREDONA, 1989).

Pero las intervenciones antrópicas implican, además, serios problemas de inestabilización de los taludes (como los señalados en Alcoi), no sólo por ocupar dichas unidades morfológicas, sino también porque en otras que no presentan un grado de peligrosidad tan elevado, la alteración del perfil de los taludes por la realización de terrazas de cultivo o el trazado de vías de comunicación, determina la inestabilización de los mismos y el desarrollo acelerado de deslizamientos y movimientos de masa, evidenciados en grietas de despegue dispuestas en arcos concéntricos, cuya concavidad se orienta hacia el frente del talud margoso.

Hay que diferenciar pues dos tipos de situaciones: la primera derivada de la carencia de una valoración adecuada de este tipo de unidades morfológicas, es decir, la ocupación de un espacio con un riesgo evidente de deslizamiento; y, la segunda, relacionada con las modificaciones y alteraciones introducidas a través de las actuaciones antrópicas, o sea, los «impactos» que provocan la inestabilización de los terrenos margosos, cuya previsión hubiera supuesto la adopción de medidas correctoras en el tratamiento de los taludes margosos resultantes.

\section{A modo de conclusión}

En definitiva, se ha tratado de demostrar que, de alguna manera, los ecosistemas presentan unos límites de tolerancia de acuerdo con la intensidad y frecuencia de las intervenciones antrópicas y de la consiguiente capacidad de alterar su dinámica (TRICART, 1982). Se trata, pues, de definir una serie de unidades morfológicas a las que les es condigna una dinámica que, en sí, puede entrañas cierta peligrosidad — riesgo potencial— ; pero que, a su vez, cada una de estas unidades, presenta también un grado de vulnerabilidad ante determinadas intervenciones antrópicas — «impactos»-, y, estas últimas pueden determinar la actualización de un problema que era latente: riesgo potencial. Es, en suma, un planteamiento general o forma de acometer un diagnóstico del territorio, en el que resulta básico el conocimiento no sólo del comportamiento de cada unidad (frecuencia, intensidad y distribución de un fenómeno), sino también los efectos de las intervenciones antrópicas.

Todo ello constituye un sistema de valoración territorial que puede resultar de gran interés en la definición de la capacidad de acogida del territorio, cuyo ámbito de aplicación más directo sería el planeamiento tanto a escala municipal: mediante una perfecta integración de la información urbanística de Planes Generales de Ordenación Urbana y Normas Subsidiarias en el desarrollo de dichos documentos, es decir, conferir a la información territorial el carácter de estudios de acogida del territorio, como a escala supramunicipal: a 
través de la aprobación de Planes Especiales de Protección del Medio Físico; aspectos que, no por evidentes, resultan frecuentes en la práctica. De hecho, la carencia de este tipo de estudios de base ha determinando que, en la actualidad, buena parte de los problemas queden reflejados, cuando no omitidos deliberadamente, en las Evaluaciones de Impacto Ambiental (E.I.A.), a todas luces insuficientes e inoperantes en este tipo de cuestiones.

\section{Bibliografía}

CALVO GARCÍA-TORNEL, F.: «La Geografía de los riesgos», Geo-crítica, n. ${ }^{\circ}$ 54, 1984.

COSTA MÁS, J. y MATARREDONA COLL, E.: «Avenidas y problemas de taludes en la ciudad de Alcoi», en GIL OLCINA, A. y MORALES GIL, A. (edit.): Avenidas fluviales e inundaciones en la cuenca del Mediterráneo, Instituto Universitario de Geografía, Universidad de Alicante, 1989. GIL OLCINA, A. et al.: Lluvias torrenciales e inundaciones en Alicante, Instituto Universitario de Geografía, Universidad de Alicante, 1983.

GIL OLCINA, A. et al.: Inundaciones en la ciudad y término de Alicante, Universidad de AlicanteAyuntamiento de Alicante, 1986.

HARVEY, A.: «The role of piping in the development of badlands and gully systems in south-east Spain», in BRYAR, R. and YAIR, R. (edit.): Badland Geomorphology and Piping, Geo Books, Norwich, 1982.

LA ROCA, N.: Evolución de laderas en la montaña meridional valenciana, Tesis Doctoral, Universidad de Valencia, 1990.

MARCO MOLINA, J. A.: Aitana. Análisis morfoestructural, Instituto Universitario de Geografía, Universidad de Alicante e Instituto de Cultura «Juan Gil-Albert», Diputación de Alicante, 1990.

MORALES GIL, A.: «El riego con aguas de avenidas en las laderas subáridas», Papeles del Departamento de Geografía de Murcia, I, 1969.

- «Abandono y desorganización de los sistemas tradicionales de riego de turbias. Su incidencia en la escorrentía», Los paisajes del agua, Universidades de Valencia y Alicante, 1989.

MORALES GIL, A.; BRU RONDA, C. y BOX AMORÓS, M.: «Las avenidas en los barrancos de las Ovejas y del Agua Amarga. Alicante, octubre de 1982», Estudios Geográficos, n.ㅇ 170-171, Madrid, 1983.

MORALES GIL, A. y BOX AMORÓS, M.: «El aprovechamiento del agua y los suelos en un dominio semiárido: la cuenca del barranco Blanco. Agost (Alicante)», Investigaciones Geográficas, n. ${ }^{\circ}$ 4, Instituto Universitario de Geografía, Universidad de Alicante, 1986.

NEBOIT, R.: L'homme et l'erosion, Ass. des Publ. Fac. des Lettres et Sciences Humaines de Clermont-Ferrand, 1983.

TRICART, J. y KILLIAN, A.: La eco-geografía y la ordenación del medio natural, 1982.

VERA REBOLLO, J. F. y MARCO MOLINA, J. A.: «Impacto de los usos del suelo y erosión en cuencas vertientes del sur del País Valenciano», Investigaciones Geográficas, n. ${ }^{\circ}$ 6, Instituto Universitario de Geografía, Universidad de Alicante, 1988. 